\title{
INSTABILITY OF THE CONSERVATIVE PROPERTY UNDER QUASI-ISOMETRIES
}

\author{
TERRY LYONS
}

\begin{abstract}
The paper shows by example the existence of a pair of quasi-isometrically equivalent complete Riemannian metrics $g, \tilde{g}$ on a manifold $N$ so that $(N, g)$ is conservative while $(N, \tilde{g})$ is not. This contrasts with known sufficient criteria for conservativeness which are stable under such changes in metric. In doing so we also give an example of a complete manifold with no nonconstant bounded solutions to Laplace's equation but with nonconstant bounded solutions to the heat equation.
\end{abstract}

\section{Introduction}

A smooth Riemannian manifold $(N, g)$ has associated with it a number of standard and interrelated geometric objects. In particular, it possesses a unique geodesic through each point in each tangent direction, a Laplace Beltrami operator, and a Markovian family $\left(\mathbf{P}_{n}, n \in N\right)$ of measures on the continuous paths called Brownian motion. The measures $\mathbf{P}_{n}$ can be constructed as the limit where $\varepsilon \rightarrow 0$ of the simple random walk one obtains by: starting at $n$, using the metric to choose a direction uniformly at random, moving at speed $\frac{1}{\varepsilon}$ along the geodesic a distance $\sqrt{\varepsilon}$, and then repeating the process with this new point as starting point. The law of this process is a probability measure on paths in $N$; the weak limit $\mathbf{P}_{n}$ is Brownian motion. The semigroup $P_{t}$ defined by $P_{t} f(n)=\mathbf{E}_{n}\left(f\left(X_{t}\right)\right)$ has $\frac{1}{2} \Delta$ as the infinitesimal generator, where $\mathbf{E}_{n}$ is the expectation or integral against $\mathbf{P}_{n}$.

As the example of the usual disc $D=\{|z|<1\} \subset \mathbf{C}$ shows, geodesics do not need to stay in $N$ for all $t$ and might explode (that is, eventually leave every compact set) in finite time. It is a well-known piece of analysis that any geodesic can be extended either until it explodes or for all time; oscilliatory discontinuities do not occur. If all geodesics extend for all

Received June 19, 1990. 
time we say $N$ is complete, and this definition is compatible with the usual metric space one.

In an analogous way, the example of the disc shows that Brownian motion $X_{t}$ can leave a manifold in finite time with probability one. Just as in the case of deterministic geodesics, there is a dichotomy: with probability one, either a path $X_{t}$ explodes (leaving every compact set for a last time) at time $\xi<\infty$, or it is defined as a continuous path in $N$ for all $t$ (in which case we say $\xi=\infty)$. In this case $\lim _{t \rightarrow \infty} X_{t}$ does not exist in $N$.

In the case where $\xi=\infty$ almost surely we say $(N, g)$ is stochastically complete (or conservative). This property has a number of equivalent purely analytic forms (see [1]).

The punctured complex plane illustrates that a manifold can be stochastically complete without being complete. A suitably chosen conformal and rotation invariant change of metric on the disc demonstrates that the converse can also occur. However, an unublished manuscript of Li and Karp, [4] and a more recent paper of Grigoryan [2] and preprint by Takeda [7] all give reasonably sharp (and quasi-isometry invariant) criteria for stochastic completeness of complete Riemannian manifolds. In particular, a complete Riemannian manifold will be stochastically complete if as a function of radius $r$ the volume of a ball centred on some fixed point grows more slowly than $e^{r^{\alpha}}$ for some $\alpha \leq 2$.

Varopoulos has shown [8] that a subquadratic bound on the curvature would always ensure nonexplosion. This paper is concerned with the stability of stochastic completeness.

For example, if $(N, g)$ is a complete and stochastically complete manifold and $\tilde{g}$ is a uniformly equivalent metric on $N$ (so that the supremum over all $n \in N$ and $u \in T N_{n} \backslash\{0\}$ of $|\log (g(u, u) / \tilde{g}(u, u))|$ is finite), then will $(N, \tilde{g})$ be complete and stochastically complete? The answer to the former is obviously yes because of the uniform equivalence of the metric spaces. This paper shows that $(N, \tilde{g})$ need not in general be stochastically complete.

The idea is relatively simple, the first step will be to look at a surface with two metrics $(N, g, \tilde{g})$ and understand the two diffusions $X$ and $\widetilde{X}$. The manifolds $(N, g)$ and $(N, \tilde{g})$ will actually both have constant negative curvature -1 and locally uniform geometries. Neither $X$ nor $\widetilde{X}$ explodes.

The second stage will be to introduce a conformal change to the metrics $h=s g$ and $\tilde{h}=s \tilde{g}$, where $s$ is a positive scalar function. Of course $h$ and $\tilde{h}$ are equivalent if $g$ and $\tilde{g}$ were, and because $(N, g)$ is a Riemannian surface the new Brownian motion $Y$ on $(N, h)$ is a simple 
time change of the old one. Put

$$
\tau(t)=\int_{0}^{t} s\left(X_{t^{\prime}}\right) d t^{\prime} \text { and } Y_{\tau}=X_{t}
$$

then Levy observed that $Y$ is Brownian motion on $(N, h)$ if $X$ was Brownian motion on $(N, g)$. Clearly $(N, h)$ will be stochastically complete if and only if $\int_{0}^{\infty} s\left(X_{t^{\prime}}\right) d t^{\prime}=\infty$.

In what follows we will generally use hyperbolic two-space [6] as a reference manifold which will induce geometries on other manifolds, and it would be possible to construct a suitable $s$ directly on $\mathbf{H}$. However, it will be conceptually easier to work on a manifold which "looks like" the Caley graph of the free group $\Gamma$ on two generators-as the tree structure will simplify matters. Most of the technical work has already been done in [5].

\section{The two metrics on $N$}

Consider $\Gamma$, the free group generated by the examples $a, b$ without relations. Any element $\gamma \in \Gamma$ has a unique shortest expression as a product of the generators $a, b$ and their inverses $a^{-1}, b^{-1}$ called the reduced word for $\gamma$. Let $|\gamma|$ denote the length of this reduced word, with $|\gamma|_{a}$ the number of $a^{ \pm 1}$, s and $|\gamma|_{b}$ the number of $b^{ \pm 1}$ 's in it. Clearly $|\gamma|=$ $|\gamma|_{a}+|\gamma|_{b}$.

Introduce a family of reversible random walks on $\Gamma$ defined by

$$
\mathbf{P}^{p}\left(X_{n+1}=g X_{n}\right)= \begin{cases}p / 2 & \text { if } g=a^{ \pm 1} \\ (1-p) / 2 & \text { if } g=b^{ \pm 1} \\ 0 & \text { otherwise }\end{cases}
$$

where $p \in(0,1)$ parameterizes the family.

Let $G$ be the Caley graph with vertex set $\Gamma$, so $\gamma$ is connected to $\gamma^{\prime}$ if $\left|\gamma^{\prime} \gamma^{-1}\right|=1$; the edge is an " $a$ " edge if $\gamma^{\prime} \gamma^{-1} \in\left\{a, a^{-1}\right\}$, otherwise it is a " $b$ " edge. Attach to each $a$-edge the weight $w_{\gamma \gamma^{\prime}}=p$ and each $b$-edge the weight $w_{\gamma \gamma^{\prime}}=1-p$. Then, if $p, p^{\prime}$ come from a compact subset of $(0,1)$ we have $\frac{1}{c}<w_{\gamma \gamma^{\prime}}^{p} / w_{\gamma \gamma^{\prime}}^{p^{\prime}}<c$ uniformly over edges $\gamma \gamma^{\prime}$. The weights can be used in the usual way to construct $\mathbf{P}^{p}$ as a reversible Markov chain in the usual way (see $[5, \S 1]$ ).

As $X$ progresses, the $\mathbf{P}^{p}$-probability that $\left|X_{n+1}\right|-\left|X_{n}\right|=+1$ is at least $\rho=\min [(1-p / 2), 1-((1-p) / 2)]$, where $\rho>\frac{1}{2}$, otherwise 
||$X_{n+1}|-| X_{n}||<1$. Thus, $\lim _{n \rightarrow \infty}\left|X_{n}\right| / n \geq\left(\rho-\frac{1}{2}\right)$, and in fact the Kingman subadditive ergodic theorem [3] implies that $n^{-1}\left|X_{n}\right|_{a}$ and $n^{-1}\left|X_{n}\right|_{b}$ converge as $n$ goes to $\infty$.

In $[5, \S 3]$ this author demonstrated that $\varepsilon$ and $\delta$ exist so that if $p<\varepsilon$

$$
\lim _{n \rightarrow \infty} \frac{\left|X_{n}\right|_{a}}{\left|X_{n}\right|}<\frac{1}{2+\varepsilon}
$$

and conversely.

In $[5, \S \S 4,5]$ it was also explained how to construct a manifold $L$ which was a spheres and tubes approximation to the graph $G$, and introduce a family of conformal structures on $K$ so that any compatible metric $g^{p}$ and associated Brownian motion $Z_{t}^{p}$ on $L$ had the property that each time when it went from a sphere $S_{\gamma}$ to another sphere $S_{\gamma^{\prime}},\left|\gamma^{\prime} \gamma^{-1}\right|=1$, and moreover the following hold:

(i) $\mathbf{P}\left(\left|\gamma^{\prime}\right|>|\gamma|\right)>\rho>\frac{1}{2}$ uniformly in $\gamma$.

(ii) Denote that part of $N$ made up of spheres $S_{\gamma}$ with $|\gamma|_{a}>$ $(1+\varepsilon)\left|\gamma_{b}\right|$ together with adjoining tubes by $A$. Then $A$ is an absorbing set for the diffusion $Z_{t}^{p}$ whenever $p$ is close enough to 1 .

(iii) $\Gamma$ acts in a natural way on $L$, and all the conformal structures on $L$ lifted from $L / \Gamma$ are invariant.

In fact, $L / \Gamma$ can be taken to be the sphere with two handles, and $g_{p}$ is the lift of the canonical hyperbolic metric on $K$.

Now the ergodic theorem for Brownian motion on the compact manifold $K$ guarantees that the number of crossings $Z_{t}$ makes from one sphere $S_{\gamma}$ to an adjacent one $S_{\gamma^{\prime}}$ is approximately linear in time, and so we can deduce that $\lim _{t \rightarrow \infty} d^{p}\left(Z_{0}^{p}, Z_{t}^{p}\right) / t>\left(\rho \cdot \frac{1}{2}\right)>0$, where $d$ is the Riemannian distance. If one lifts the process up the universal cover $\mathbf{H}$, one obtains ordinary hyperbolic Brownian motion $Z^{h}$. Standard properties of Brownian motion there ensure the inequality

$$
\varlimsup_{T \rightarrow \infty} \frac{d\left(Z_{T}^{h}, Z_{0}^{h}\right)}{T}<\infty,
$$

where $d$ is hyperbolic distance, and $Z^{h}$ (the lift of $Z^{p}$ ) is hyperbolic Brownian motion (see, for example, [6]).

\section{The conformal transformation}

Consider $L$ a bit further. Let $g, \tilde{g}$ be two metrics on $L$ corresponding to $g^{p}$ for $p$ close to zero, and again for $p$ close to 1 . Because $g, \tilde{g}$ are 
defined on $L / \Gamma=K$ and $K$ is compact, they are uniformly equivalent. Clearly they are complete metrics, for let $\rho(t)$ be any geodesic in $(L, g)$ that explodes; the only way it can do this is by visiting $\infty$-many spheres. Let $S_{\gamma_{1}} S_{\gamma_{2}}, \cdots$ be the sequence of distinct spheres visited. Then $\left|\gamma_{j} \gamma_{j-1}^{-1}\right|=$ 1 for all $j$, and the minimum distance from one sphere $S_{\gamma}$ to one of four neighbours is some strictly positive $\varepsilon$, independent of $\gamma$ so the length of the geodesic $\rho$ is at least $n \varepsilon$ by the time it has visited the $n$th sphere in the sequence, and thus has length infinity. As it travels at unit speed it is defined for all time.

Which conformal changes of metric preserve the completeness? Consider $(L, s g)$; suppose that $s$ is slowly varying in that there is a $C>1$ such that uniformly in $\gamma$ if $x, x^{\prime}$ are in $S_{\gamma}$, the directly neighbouring spheres $S_{\gamma^{\prime}}$, or the connecting cylinders, then

$$
\frac{1}{C}<\frac{s(x)}{s\left(x^{\prime}\right)}<C .
$$

Fix $0 \in S_{e}$, and let $x_{\gamma}=\gamma 0$; then

$$
d_{s g}\left(S_{\gamma}, S_{\gamma^{\prime}}\right) \geq\left[\frac{S\left(x_{\gamma}\right)}{C}\right]^{1 / 2} d_{g}\left(S_{\gamma}, S_{\gamma^{\prime}}\right),
$$

whenever $\gamma, \gamma^{\prime}$ are adjacent.

Lemma. Suppose $s$ is slowly varying and $s\left(x_{\gamma}\right)>\psi(|\gamma|)$, where $\sum_{n=1}^{\infty}[\psi(n)]^{1 / 2}=\infty$; then $(L, s g)$ is complete.

The proof is as before. Any geodesic going to infinity must for some $N$ and for each $n>N$ pass from some $S_{\gamma}$ to $S_{\gamma^{\prime}}$ with $|\gamma|=n$ and $\left|\gamma \gamma^{\prime-1}\right|=1$. It does this in distinct time intervals. It follows that a lower bound for its length is $C^{-1 / 2} \sum_{N}^{\infty} \psi(n)^{1 / 2}$. q.e.d.

In particular, it suffices that $s(x) \sim d_{g}(0, x)^{\alpha}$ for any $\alpha \geq-2$, for the space to be complete.

Now what sort of choice for $s$ will ensure stochastic (in)completeness? One uses the fact that $d_{g}\left(0, Z_{t}\right) \sim t$ to see that $\int_{0}^{\infty} s\left(Z_{t}\right) d t<\infty$ if $s(x) \sim d_{g}(0, x)^{\beta}$, where $\beta<-1$, and $\int_{0}^{\infty} s\left(Z_{t}\right) d t=\infty$ if $\beta \geq-1$.

Lemma. Let $A \subset L$ be an absorbing set for $Z$ and suppose that on $A$

(i) $s(x) \sim d(0, x)^{\beta}, \beta \geq-1$, or

(ii) $s(x) \sim d(0, x)^{\beta}, \beta<-1$.

Then $(L, s g)$ is stochastically (i) complete or (ii) incomplete. 
Proof. The divergence or convergence of $\int_{0}^{\infty} s\left(X_{t}\right) d t$ only depends on what happens near $\infty$, and as $X_{t} \in A$ for all large $t, s\left(X_{t}\right) \sim t^{\beta}$ for all large $t$.

\section{The final example}

It is now absolutely straightforward to construct the complete example.

Let $A \subset L$ be the union of those spheres $S_{\gamma}$ with their joining tubes for which $|\gamma|_{a}>\left(\frac{1}{2}+\varepsilon\right)|\gamma|$, and let $B$ be the similar region where $|\gamma|_{b}>$ $\left(\frac{1}{2}+\varepsilon\right)|\gamma| . A$ is absorbing for $Z_{t}, B$ for $\tilde{Z}_{t}$. If $s-d(0, x)^{-\beta}$ on $A$ and $s \sim 1$ on $B$, where $1<\beta \leq 2$, then $(L, g)$ and $(L, \tilde{g})$ are both complete, however only $(L, \tilde{g})$ is stochastically complete. A suitable choice for $s$ would be some interpolation of the function which at $x_{\gamma}$ has the value

$$
\left\{\left(|\gamma|_{a}-|\gamma|_{b}\right)^{+}+1\right\}^{-\beta}, \quad \beta \in(1,2],
$$

and in fact $p=\frac{1}{2}$ would be the critical point in this case. No one has investigated the behaviour at this point as $\beta$ changes.

Final remark. A number of years ago a colleague asked if one could find a complete manifold with no nonconstant bounded (positive) harmonic functions but lots of bounded solutions to the heat equation; at that time this author could not. The approach here provides an example. In $[5, \S 6]$ we constructed a more complex manifold modelled on the free group on two generators which had no positive harmonic functions. The same conformal metric change can be applied there; and precisely because the extra links were chosen to connect only words with the same proportion of $a$ 's and $b$ 's one discovers that the same function $s$ will introduce a conformal change of metric which preserves completeness but forces explosion. Explosion gives a very strong existence results for globally defined bounded nonconstant solutions to the heat equation on $L \times \mathbf{R}_{+}$.

\section{References}

[1] E. B. Davies, $L^{1}$ properties of second order operators, Bull. London Math. Soc. 17 (1985) 417-436.

[2] A. A. Grigoryan, On stochastically complete manifolds, Soviet Math. Dokl. 34 (1987) 310-313.

[3] J. C. F. Kingman, Subadditive processes, (Ecole d'Ete Probab. Saint Flour 1975), Lecture Notes in Math., Vol. 539, Springer, Berlin, 1976.

[4] P. Li \& L. Karp, The heat equation on complete Riemannian manifolds, unpublished manuscript.

[5] T. J. Lyons, Instability of the Liouville property for quasi-invariant Riemannian manifolds and reversible Markov chains, J. Differential Geometry 26 (1987) 33-66. 
[6] _ _ A synthetic proof of Makarov's law of the iterated logarithm, Bull. London Math. Soc. 22 (1990) 159-162.

[7] M. Takeda, On the conservativeness of the Brownian motion on a Riemannian manifold, preprint.

[8] N. Varopoulos, Brownian motion and random walks on manifolds, Ann. Inst. Fourier (Grenoble) 34 (1984) 243-269.

UNIVERSITY OF EDINBURGH 
